
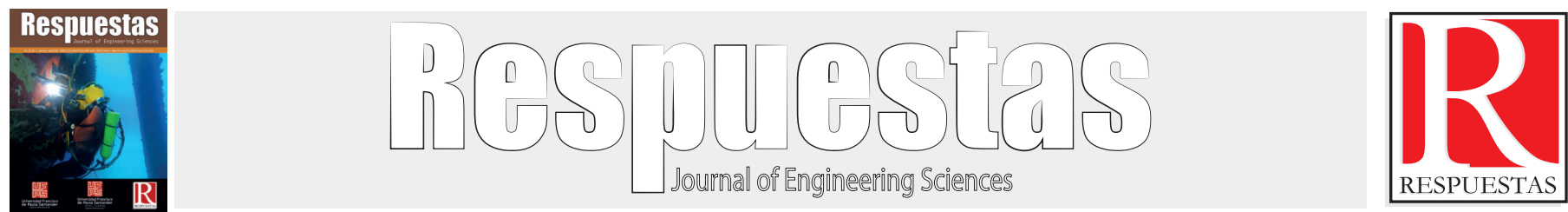

Original Article

https://doi.org/10.22463/0122820X.2405

\title{
Analysis of the Transverse Force in the Rayleigh and Mie Approximations for a Capture
} Beam TEM ${ }_{00}$ and TEM ${ }_{01}^{*}$

Análisis de la fuerza transversal en las aproximaciones de Rayleigh y Mie para un haz de captura TEM $\mathrm{T}_{00} \mathrm{y}$ TEM $^{*}$

Darby Páez Amaya ${ }^{1}$, Frederic Català Castro ${ }^{2}$, Martha Lucia Molina Prado ${ }^{3 *}$, Mario Montes-Usateguii ${ }^{4}$ Néstor Alónso Arias Hernández $^{5}$

${ }^{\prime}$ Magister en Fisica, darbypaezamaya@gmail.com, Orcid: 0000-0002-2204-5344, Universidad de Pamplona, Pamplona, Colombia.

2Doctor en Nanociencias, frederic.catala@icfo.eu, Orcid: 0000-0003-3704-1861, Universitat de Barcelona, Barcelona, Spain.

3*Doctor en Ciencias Naturales-Física, marlumopra@unipamplona.edu.co, Orcid: 0000-0002-3347-7986, Universidad de Pamplona, Pamplona, Colombia.

${ }^{4}$ Doctor en Física, mario montes@ub.edu.es, 0000-0003-2811-1485, Universitat de Barcelona, Barcelona, Spain.

'Doctor en Ciencias Naturales-Física, nesariher@unipamplona.edu.co, Orcid: 0000-0002-9224-7654, Universidad de Pamplona, Pamplona, Colombia.

How to cite: D. Páez-Amaya, F. Català-Castro, M.L Molina-Prado, M. Montes-Usategui, N.A. Arias-Hernández. "Analysis of the Transverse Force in the Rayleigh and Mie Approximations for a Capture Beam TEM ${ }_{00}$ and TEM ${ }_{01}^{*}$ ". Respuestas, vol. 25, no. 1, pp. 53-59, 2020.

Received on July 28, 2019; Approved on November 13, 2019

\begin{tabular}{ll}
\hline Keywords: & ABSTRACT \\
\hline & Optical tweezers use a highly-focused laser beam to capture and manipulate micro- and nanometric objects. These have \\
been demonstrated to be a promising devices for state-of-the-art research in several fields, such as microbiology and \\
biophysics. The prediction of the optical forces that are present in this phenomenon is a current problem in continuous \\
laser trapping, \\
evolution. Additionally, the recent use of vortex beams with exotic properties as the orbital angular momentum, with \\
advantages as the rotational manipulation of the captured microparticles and reduction of the optical damage in biological \\
samples (Opticution), makes the problem even more complex. Mathematical expressions in the Rayleigh and Mie regimes \\
for the radiation force on a dielectric sphere captured by TEM ${ }_{00}$ and TEM ${ }_{01}^{*}$ mode beams are presented. Theoretical results \\
are then compared with experimental measurements obtained with a direct force measurement device based on light- \\
momentum detection.
\end{tabular}

\begin{tabular}{|c|c|}
\hline Palabras clave: & RESUMEN \\
\hline $\begin{array}{l}\text { Pinzas ópticas, } \\
\text { trampas láser, } \\
\text { rayos vórtex. }\end{array}$ & $\begin{array}{l}\text { Las pinzas ópticas utilizan un rayo láser altamente enfocado para capturar y manipular objetos micro y } \\
\text { nanométricos. Se ha demostrado que son un dispositivo prometedor para la investigación de vanguardia en } \\
\text { varios campos, como la microbiología y la biofísica. La predicción de las fuerzas ópticas presentes en este } \\
\text { fenómeno es un problema actual en continua evolución. Además, el reciente uso de haces de vórtice con } \\
\text { propiedades exóticas como el momento angular orbital, con ventajas como la manipulación rotacional de las } \\
\text { micropartículas capturadas y la reducción del daño óptico en las muestras biológicas (Opticution), hace que } \\
\text { el problema sea aún más complejo. Se presentan expresiones matemáticas en los regímenes de Rayleigh y } \\
\text { Mie para la fuerza de radiación sobre una esfera dieléctrica capturada por los haces de los modos TEM }{ }_{00} \text { y } \\
\text { TEM }^{*} \text {. A continuación, se comparan los resultados teóricos con las mediciones experimentales obtenidas con } \\
\text { un dispositivo de medición directa de la fuerza basado en la detección del momento de la luz. }\end{array}$ \\
\hline
\end{tabular}

\section{Introduction}

The invention of Optical Tweezers has been followed by a wide number of research applications in several fields, such as Atomic Physics [2], Nanotechnology [3], Colloidal Sciences [4], and Fundamental Physic [5]. Among these, OT has become especially promising in several topics in biophysics, e.g. microbiology and cell mechanics, thanks to the non-invasive principle of OT. Forces in the range of $0.1-1000 \mathrm{pN}$ are precisely accessible with calibrated OTs, which makes them versatile tools in experiments in vivo.

*Corresponding author:

E-mail Address:agonzale@saber.uis.edu.co (Octavio Andrés González Estrada)

(c) (i) $\risingdotseq$ Peer review is the responsibility of the Universidad Francisco de Paula Santander. (c) $(9)$
By NC ND
Interestingly, trapping beams with angular momentum have been increasingly used by the optical trapping community, with a wide number of applications [6] - [8]. Compared to regular Gaussian beams, doughnut-shaped modes, such as $\mathrm{TEM}_{01}^{*}$, are suitable to avoid laserinduced heating and optical damage (opticution) [9], since the intensity profile drops to zero at the optical axis. In this work, we compare optical force measurements using a force sensor (Lunam $^{\mathrm{TM}} \mathrm{T}-40 \mathrm{i}$, Impetux Optics, Spain) on dielectric microspheres with the theoretical profiles obtained in Rayleigh and Mie regimes. 


\section{Materials and methods}

Calculation of optical forces can be performed analytically for particles far smaller and larger compared to the trapping beam wavelength. In the first case, the Rayleigh regime (lambda $>>$ D) one can apply Rayleigh's Dipolar Theory (RDT). In the second case, the Mie regime (lambda $<<$ D), Optical Ray Theory (ROT) can be applied to compute optical force.

\section{Rayleigh Regime}

This method is applicable if the radius of the captured sphere is smaller than the wavelength of the trapping beam. Some research papers state that, for this method, "a $<\lambda / 20 "$ [12], and it consists in considering light as electromagnetic waves. Then it is possible to analyze the behavior of the radiation sphere when it interacts with the capture radiation. As the size of the sphere is small, the electromagnetic field incident on the sphere can be considered homogeneous, allowing the dielectric sphere to behave as an electrical point-like dipole.

In our work, we are interested in the transverse force. Using this method, the transverse force is consequence of the interaction of the electric and magnetic fields of the light beam on the sphere and is exactly the Lorentz's force. In order to find an expression for this force, it is necessary to calculate the electric and magnetic force on the sphere [10], and using the correct math form of the electric field of beam capture. Substituting these math expressions of $\mathrm{TEM}_{00}$ mode it is possible to define it on this way $[10,13]$,

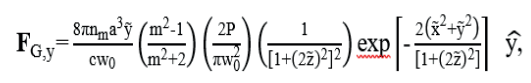

And $\mathrm{TEM}_{01}^{*}$ mode $[12,14,15]$

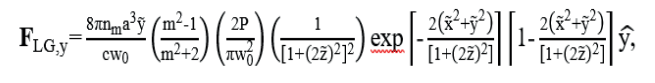

Where, $(\tilde{x}, \tilde{y}, \tilde{z})=\left(\mathrm{x} / \mathrm{w}_{0}, \mathrm{y} / \mathrm{w}_{0}, \mathrm{z} /\left(\mathrm{kw}_{0}^{2}\right)\right.$ are the three normal coordinates, $\mathrm{k}$ is the wave number in the medium, $\mathrm{P}$ is the power of the laser beam and $\mathrm{w}_{0}$ is the radius of the beam waist, $a$ is the radius of sphere, $m=n_{p} / n_{m}$ is the refractive index of the sphere $\left(\mathrm{n}_{\mathrm{p}}\right)$ relative to environment $\left(\mathrm{n}_{\mathrm{m}}\right)$ and $\mathrm{c}$ is the light velocity in vacuum.

\section{Mie Regime}

This method, so-called Optical Ray Method, is based on Geometrical Optics. It is only valid when the dimension of the trapped object is much larger than the wavelength -according to some research papers, the diameter of the sphere captured must be at least $\approx 10 \lambda[16]$. In this method is dividing the capture beam in independent light rays, in where each of complies with the Geometrical Optics approach, i.e. Snell and Fresnel laws [11, 14]. Some requirements of this method are that the captured sphere be dielectric and homogeneous. Therefore, radiation is not absorbed and the trajectory of the light rays only changes in direction when they are refracted and reflected at the surface of the dielectric sphere.

The rate of linear momentum that each light ray transfers to the trapped sphere, for the $\mathrm{TEM}_{00}$ case can be expressed as follows [14-16]:

$$
\mathbf{F}_{\mathrm{G}, \mathrm{y}}=\frac{\mathrm{n}_{\mathrm{m}} \mathrm{P}}{\mathrm{c}}\left[\frac{8}{\pi w_{0}} \int_{0}^{\pi / 2} \mathrm{~d} \beta \int_{0}^{\mathrm{W}_{0}} \exp \left(-\frac{2 r^{2}}{w_{0}^{2}}\right)\left\{\mathrm{R}_{\theta} \sin -\mathrm{T}_{\theta} \mathrm{T}_{\Gamma}\left[\frac{\sin (2 \theta-2 \Gamma)+\mathrm{R}_{\Gamma} \sin (2 \theta)}{1+\mathrm{R}_{\Gamma}^{2}+2 \mathrm{R}_{\Gamma} \cos (2 \Gamma)}\right]\right\} \mathrm{dr}\right] \sin (\gamma) \hat{\mathrm{y}}
$$

And, for the $T E M_{01}^{*}$ mode:

$$
\mathbf{F}_{\mathrm{LG}, \mathrm{y}}=-\frac{\mathrm{m}_{\mathrm{m}} \mathrm{P}}{\mathrm{c}}\left[\frac{8}{\pi w_{0}^{2}} \int_{0}^{\pi / 2} \mathrm{~d} \beta \int_{0}^{w_{0}} \mathrm{r}\left(\frac{\sqrt{2}}{\mathrm{w}_{0}}\right)^{2} \exp \left(-\frac{2 \Gamma^{2}}{\mathrm{w}_{0}^{2}}\right)\left\{\mathrm{R}_{\theta} \sin -\mathrm{T}_{\theta} \mathrm{T}_{\Gamma}\left[\frac{\sin (2 \theta-2 \Gamma)+\mathrm{R}_{\Gamma} \sin (2 \theta)}{1+\mathrm{R}_{\Gamma}^{2}+2 R_{\Gamma} \cos (2 \Gamma)}\right]\right\} \mathrm{dr}\right] \sin (\gamma) \hat{\mathrm{y}} .(4)
$$

Here, $\Gamma$ is the angle of refraction, $R_{\theta}$ and $T_{\theta}$ are the Fresnel coefficients (reflectance and transmission, respectively) for a ray entering the sphere, $\mathrm{R}_{\Gamma}$ and $\mathrm{T}_{\Gamma}$ where the angle of incidence is $\Gamma$ when the ray goes from the inner to the surface of the sphere, this is showed in the Fig. 1a, $r$ and $\beta$ are the radial and azimuthal coordinates of capture beam and $\theta$ is the angle between a normal vector at surface of the sphere and the rays of light on the sphere. This $\theta$ angle must be found using the geometrical relation a $\sin (\theta)=S^{\prime} \sin (\gamma)$, where a it is the sphere radius and $\mathrm{S}^{\prime}$ it is the transversal length (axis $\mathrm{y}$ ) from the center of sphere (Figure 1b). 


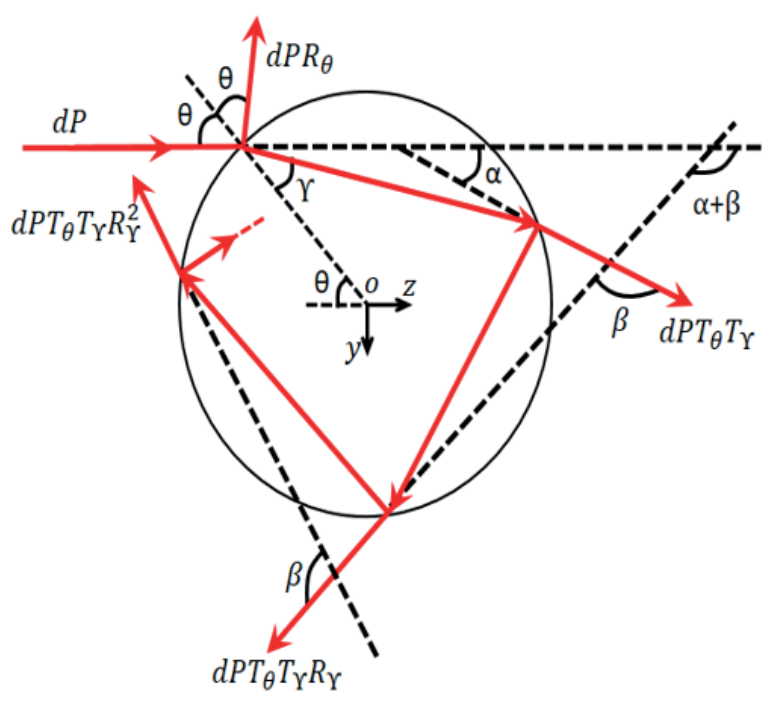

(a)

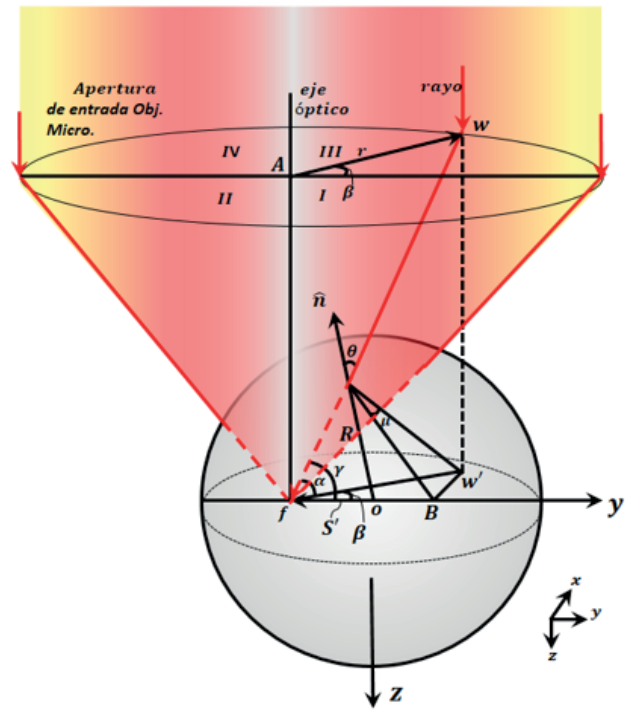

(b)

Figure 1. In (a) Geometric diagram to calculate for calculating the force due to single incident ray of power dP (b) Trap geometry with the beam focused along the -Y axis at a distance S' from the origin.

\section{Preparing the Experiment}

In order to measure the optical force, we used a holographic optical tweezers (HOTs) set-up (Figure 2a). This system uses a laser emitting a Gaussian $\mathrm{TEM}_{00}$ beam, with wavelength $\sim 1060 \mathrm{~nm}$ (IR) and adjustable power from 0 until 1 $\mathrm{W}$ and linear polarization. The optical system has initially a telescope, formed by one couple of lenses $\mathrm{L}_{1}$ and $\mathrm{L}_{2}$, and whose function is to expand the laser spot by a factor of $\mathrm{f} 1 / \mathrm{f} 2$. This magnification is necessary to fill the work area of a Liquid Crystal SLM-LCO (X10468 Hamamatsu), that is electronically controlled by a computer. After modulation of the beam, it is necessary to readjust the size of beam by the telescope formed between $\mathrm{L}_{3}$ and $\mathrm{L}_{4}$ lenses, so that it fills the input pupil of the objective (Nikon 60X, water immersion, NA = 1.2). The objective the laser beam onto the sample plane (containing polystyrene microspheres in water) to create the optical trap. The dichroic mirror (DM), reflects the IR light while it transmits the shorter, visible wavelengths. Images are acquired with a CCD camera.

To measure the optical force on a micron-sized object, we used a direct force sensor (Lunam ${ }^{\mathrm{TM}} \mathrm{T}-40 \mathrm{i}$, Impetux Optics, Spain). This device measures the optical force directly on a trapped object using the direct measurement of the change in the light momentum [17-19]. The method can measure the optical forces in in-vivo environments and irregular objects $[9,20,21]$.

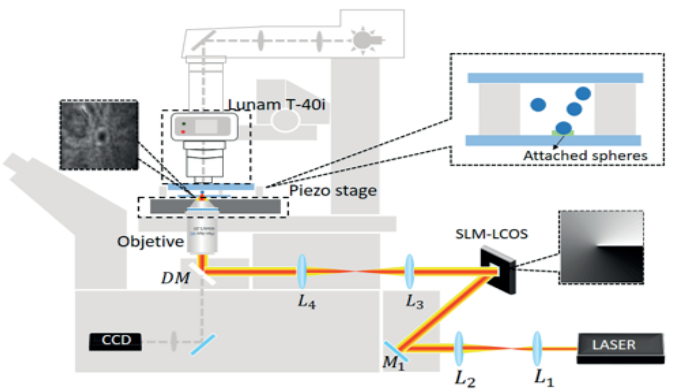

(a)

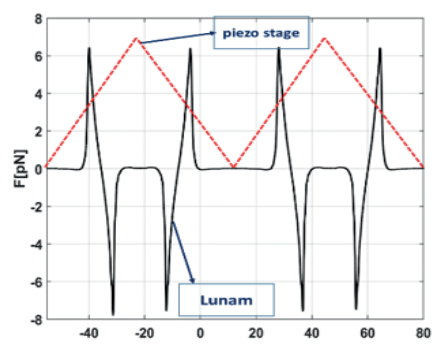

(b)

Figure 2. HOTs set-up. (a) Diagram of the optical system where $\mathrm{L}_{1}, \mathrm{~L}_{2}, \mathrm{~L}_{3}, \mathrm{~L}_{4}$ are lenses, $\mathrm{M}_{1}, \mathrm{M}_{2}$ are mirrors, DM is a dichroic mirror. (b) Force signal (black) obtained after applying a triangular oscillation with the piezo-electric stage. 
Samples for our experiments contained $\mathrm{R}=4 \mu \mathrm{m}$ m polystyrene microspheres (refractive index $\mathrm{n} \sim 1$. 6), diluted in distilled water ( $\mathrm{n} \sim 1.33$ ). After a few minutes, the microspheres deposited on the lower coverslip and attached to it. Once this was achieved, the light was focused with the microscope objective on one sphere and the sample was moved with a piezo stage (NV403CLE, PiezoSystemJena), oscillating with a triangular shape with an amplitude of $17 \mu \mathrm{m}$ of and1Hz. The force sensor was above the sample (Figure 2a), so that it was possible to obtain experimental force profile measurements with a sampling frequency of $10 \mathrm{kHz}$.

\section{Results and discussion}

We obtained transverse force profile measurements on $\mathrm{R}=4 \mu \mathrm{m}$ spheres with $\mathrm{TEM}_{00}$ and $\mathrm{TEM}_{01}^{*}$ mode trapping beams. In this process, the beam was highly focused at the axial equilibrium point and the trap power registered by the sensor was $10.37 \mathrm{~mW}$. In Figure 3a, the curve represents the optical force that the sphere experiences with respect to the trap focus. Therefore, these measurements correspond the data processing of two seconds recorded by the sensor, this correspond two oscillations of the piezo stage with triangular signal, or in other words this curve represent four experimental profiles of force on the sphere.

We next compared the measurements with the optical force calculated through the Rayleigh and Mie approximations (1-4). The theoretical profiles have been calculated with the real parameters of the experiment: refractive index of the sphere and the medium, the radius of the sphere, the trap power on the sphere monitored by the sensor, laser wavelength and beam shape $\left(\mathrm{TEM}_{00}\right)$. In Figure $3 \mathrm{~b}$, we compare the experimental result with the theoretical profile in Rayleigh regime. The curves differ by $\sigma_{\mathrm{s}}=2.7701 \mathrm{pN}$ - corresponding to $36.5 \%$ with respect to the maximum force $(7.59 \mathrm{pN})$. In this case, the difference appears because the validity condition of method $(\mathrm{R}>\lambda)$ is not met.

In the same way, in Figure 3c, we can observe the comparison between the experimental profile and the prediction in the Mie regime. The diameter of the sphere is now closer to the validity condition of the approach. These curves are different by $\sigma_{\mathrm{s}}=1.6016 \mathrm{pN}$ - corresponding to $21.1 \%$ with respect to the maximum force. This discrepancy is due to the fact that the method cannot model fully the environment of the experiment. On one hand, the beam has been considered to be described by a pure Gaussian, TEM $_{00}$ beam. On the other, the Optical Ray Method fails noticeably when the trap is focused near the surface of the trapped objects $(y \sim R)$, what may explain the observation of the experimental maximum force diverging from theory. As discussed in [22], this is mainly due to the trap focus being infinitesimal in geometrical optics.

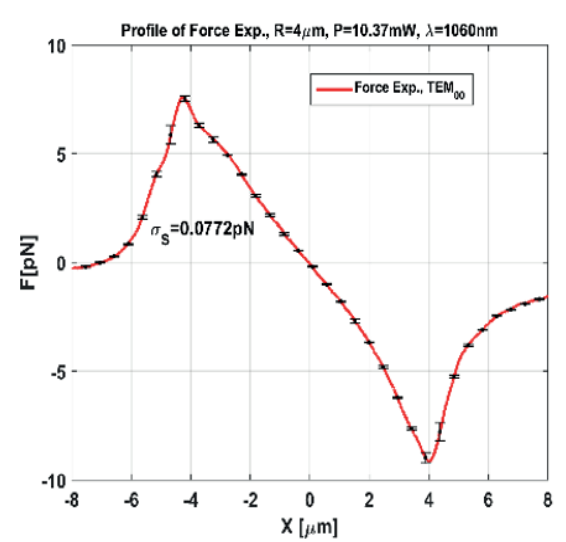

(a)

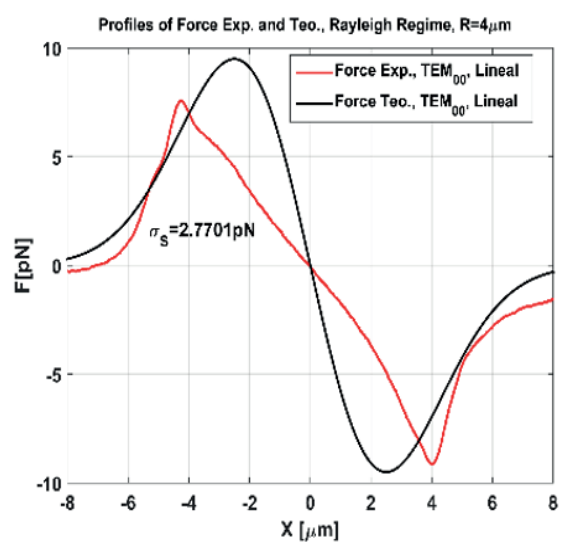

(b)

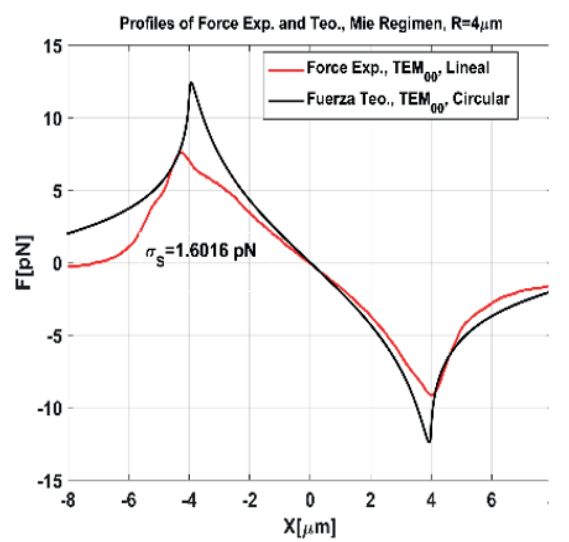

(c)

Figure 3. Force profile in a $\mathrm{TEM}_{00}$ beam. (a) Transverse optical force profile on a $\mathrm{R}=4 \mu \mathrm{m}$ sphere. (b) Comparison between the experimental profile and the theoretical profile in the Rayleigh regime. (c) Comparison with the Mie regime 
As described in Section 3, we used an SLN to generate a phase mask with helical shape of topological charge $1=1$. In this way, it was possible to obtain a Laguerre Gaussian TEM $^{*}{ }_{01}$ beam. We measured the optical force profile on the same sphere before (Figure 4a). Compared to the theoretical curve in the Rayleigh Regime (Figure 4b), the measurement deviates by an average $\sigma_{\mathrm{s}}=5.1317 \mathrm{pN}$ - which corresponds to $98.3 \%$ of the maximum force $(5,22 \mathrm{pN})$. It is clear that the Rayleigh approximation in this case does not describe the qualitative behavior of the measurement, which even exhibits a trapping stiffness (at $y \sim 0$ ) of opposite sign. This is due to the fact that the gradient force that arises after the interaction of the point-like dipole with the optical field has a substantially different origin in our experimental case. For the $\mathrm{R}=4 \mu \mathrm{m}$ microspheres used here, the trapping force is properly described in the Mie regime (Figure 4c). In this case, the curves deviate each other by $\sigma_{\mathrm{s}}=1.4219$ - according to $27.2 \%$ of the maximum force.

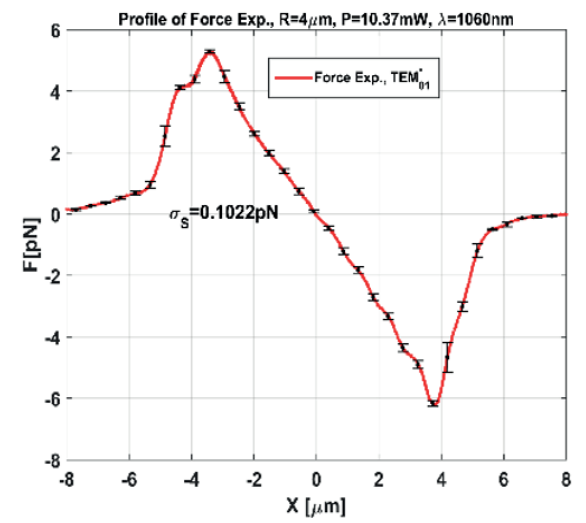

(a)

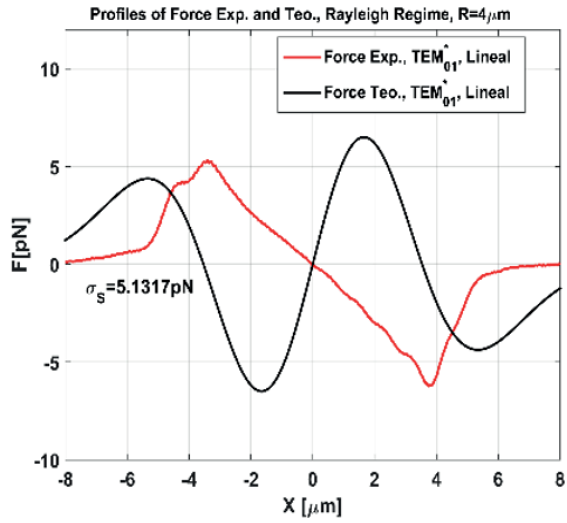

(b)

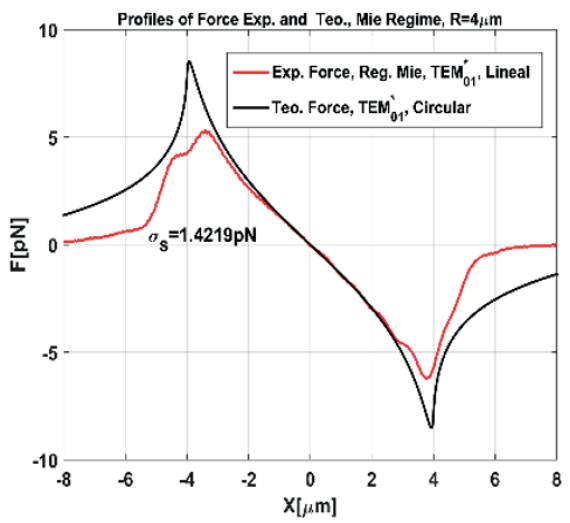

(c)

Figure 4. Force profile in a $\mathrm{TEM}^{*}{ }_{01}$ beam. (a) Transverse optical force profile on a $\mathrm{R}=4 \mu \mathrm{m}$ sphere. (b) Comparison between the experimental profile and the theoretical profile in the Rayleigh regime. (c) Comparison with the Mie regime.

\section{Conclusions}

In this work we compared experimental measurements of optical forces and the theoretical forces computed under Rayleigh and Mie regimes for $\mathrm{TEM}_{00}$ and $\mathrm{TEM}^{*}{ }_{01}$ trapping beams. Our approach consisted in using microbeads attached to a coverslip surface as force probes, which were translated across an optical trap by means of a piezo-electric stage. The optical force is well described, according to our light-momentum-based force measurements, in terms of the Mie approach, whereas the Rayleigh approximation becomes clearly far from our results, especially for the $\mathrm{TEM}_{01}^{*}$ case, due to the microspheres used being larger than the wavelength of the trapping beam.

The experimental results are described within $21 \%$ and $27 \%$ accuracy using the Mie approach for $\mathrm{TEM}_{00}$ and $\mathrm{TEM}^{*}{ }_{01}$ mode beams, respectively. We observed the experimental curves to deviate from theory, mainly, around the particle surface, i.e. $y \sim R$. A plausible reason for that phenomenon is that the trapping beam is not properly described in the trap focus, which is mathematically infinitesimal in the Optical Ray Method. Likewise, it is worth commenting that the parameters used for our simulations were exactly those reported by the manufacturer regarding the microspheres used (refractive index and size), as well as the trapping beam shape and power. For example, modifications to our calculations accounting for optical aberrations are likely to describe the experimental measurements with even closer agreement.

The theoretical Mie approach, valid for large particles, describes with proper accuracy the experimental results reported here. To conclude, the optical force profile generated by complex beams, e.g. the $\mathrm{TEM}^{*}{ }_{01}$ mode beam used here, can be precisely modeled by the Optical Ray Method and measured experimentally through light momentum detection.

\section{Acknowledgments}

The authors acknowledge the Optical Trapping Lab - Grup de Biofotònica (Barcelona, Spain), as well as 
Universidad de Pamplona (Colombia) economic support through "Movilidad Estudiantes 2018” program.

These results are part of project 400-156.012-018(GA313BP-2017-II).

\section{References}

[1] A. Ashkin, J. Dziedzic, J. Bjorkholm and S. Chu. "Observation of a single-beam gradient force optical trap for dielectric particles", Optics Letters, vol. 11, pp. 288-290, 1986.

[2] A. Ashkin, "Atomic-beam deflection by resonance-radiation pressure", Physical Review Letters, vol. 25, pp. 1321-1324, 1970.

[3] R. Agarwal, K. Ladavac, Y. Roichman, G. Yu, C. Lieber and D. Grier, "Manipulation and assembly of nanowires with holographic optical traps", Optics Express, vol. 13, pp. 8906-8912, 2005.

[4] K. Dholakia and P. Zemanek, "Colloquium: Gripped by light: Optical binding”, Reviews of Modern Physics, vol. 82, pp. 1767-1791, 2010.

[5] D. Chang, C. Regal, S. Papp, D. Wilson, J. Ye, O. Painter, H. Kimble and P. Zoller "Cavity opto-mechanics using an optically levitated nanosphere" Proceedings of the National Academy of Sciences, vol. 107, no. 3, pp.10051010, 2010

[6] R. Dasgupta, S. Ahlawat, R. Shanker, S. Shukla and P. Gupta, "Optical trapping of spermatozoa using laguerre-gaussian laser modes", Journal of Biomedical Optics, vol. 15, pp. 0650101-5, 2010.

[7] M. Padgett and R. Bowman, "Tweezers with a twist", Nature Photonics, vol. 5, pp.343-348, 2011.

[8] X. Zhou, Z. Chen, Z. Liu and J. Pu, "Experimental investigation on optical vortex tweezers for microbubble trapping", Open Physics, vol. 16. no. 5 pp. 52, 2018.

[9] C. Frederic, M. Ferran, M. Montes-Usategui, A. Farré and E. Martín-Badosa, "Influence of experimental parameters on the laser heating of an optical trap", Scientific Reports, vol. 7, no. 16052, 2017.

[10] H. Yasuhiro and A. Toshimitsu, "Radiation forces on a dielectric sphere in the Rayleigh scattering regime", Optics Communications, vol. 124, pp. 529-541, 1996.

[11] A. Ashkin, "Forces of a single-beam gradient laser trap on a dielectric sphere in the ray optics regime" Biophysical Journal, vol. 61, pp.569582, 1992.

[12] V. Garbin, D. Cojoc, R. Kulkarni, R. Malureanu, E. Ferrari, M. Nadasan and E. DiFabrizio "Numerical analysis of forces in optical tweezers in the Rayleigh regime", Proceedings of SPIE 5972, Advanced Topics in Optoelectronics, Microelectronics, and Nanotechnologies II, 597205, 2005.

[13] D. Amaya, N. Arias and M. Molina, "Interfaz Gráfica Para El Análisis De Las Fuerzas De Captura En Una Pinza Óptica Usando Las Aproximaciones De Rayleigh y Mie" Bistua: Revista de la Facultad de Ciencias Básicas, vol. 14 pp. 182-193, 2016.

[14] D. Paez, “Análisis Teórico de una Pinza óptica en las Aproximaciones de Rayleigh y Mie para un Haz de Captura Doughnut-Shaped tipo TEM $_{01}^{*}$ " tesis de Master, Universidad de Pamplona, Colombia, 2018.

[15] D. Páez, N. Arias and M. Molina, "Estudio De Las Fuerzas De Una Pinza Óptica Sobre Una Esfera Dieléctrica En El Régimen De La Óptica De Rayos Para Un Haz De Captura Laguerre Gaussiano Modo", Bistua: Revista de 
la Facultad de Ciencias Básicas, vol. 16, no. 2, pp. 3-17, 2018.

[16] I. Kang-Bin, D. Lee, K. Hyun-Ik, O. ChaHwan, S. Seok-Ho and K. Pill-Soo, "Calculation of Optical Trapping Forces on Microspheres in the Ray Optics Regime", Journal of the Korean Physical Society, vol. 40, pp. 930-933, 2002.

[17] S. Smith, Y. Cui and C. Bustamante, "Optical-trap force transducer that operates by direct measurement of light momentum", Biophotonics, vol. 361, pp. 134-162, 2003.

[18] A. Farré and M. Montes-Usategui, "A force detection technique for single-beam optical traps based on direct measurement of light momentum changes", Optics Express, vol. 18 pp. 11955$11968,2010$.

[19] A. Farré, F. Marsà and M. Montes-Usategui, "Optimized back-focal-plane interferometry directly measures forces of optically trapped particles", Optics Express, vol. 20 pp. 1227012291, 2012.

[20] J. Yonggun, K. Suvranta, R. Babu, J. Narayanareddy, K. Michelle, S. Gross, "Calibration of Optical Tweezers for in Vivo Force Measurements: How do Different Approaches Compare?", Biophysical Journal, vol. 107, no. 6, pp. 1474-1484, 2014.

[21] C. Frederic, M. Ferran, M. Montes-Usategui, A. Farré and E. Martín-Badosa, "Extending calibration-free force measurements to opticallytrapped rod-shaped samples", Scientific Reports, vol. 7, pp. 1-10, 2017.

[22] T. Nieminen, "Visual guide to optical tweezers", European Journal of Physics, vol. 38, no. 3, pp. 1-17, 2017. 